\title{
Software Reuse in Organizations: A Survey in Moroccan Software Industry Context
}

\author{
Siham Younoussi *, Ikram el Rhaffari, Mohamed Amoud, Ounsa Roudies \\ Mohammed V University in Rabat, Ecole Mohammadia d'Ingénieurs (EMI), Siweb Research Team, Morocco. \\ * Corresponding author. Email: siham.younoussi@gmail.com \\ Manuscript submitted November 29, 2018; accepted January 8, 2019. \\ doi: 10.17706/jsw.14.4.153-167
}

\begin{abstract}
A good software reuse process, contributes towards accelerating time to market of software products and reducing costs and efforts. Research raised that the adoption of software reuse is becoming a necessity for organizations to compete against others and stand out amongst them. Thereby many organizations try to invest in software reuse by identifying best reuse techniques, methods, and practices. This paper presents a complete survey method with findings backed up with statistics. The goal is to evaluate in practice the adoption of techniques, methods and practices proposed by academics, and highlight the motivations as well as the difficulties to implement a successful reuse program by different types of organizations in Morocco.

A descriptive survey of 84 software participants with different backgrounds (software managers, analysts, engineers and software developers) has been conducted, in order to identify the current state of software reuse in practice inside the Moroccan software industry. A complete survey method is presented, including the process, data collection, and analysis phases, as well as a discussion on the study's validity...The survey consisted of 28 questions, grouped into 4 sections and backed up our findings with statistics. A discussion was also given based on the comparison of the survey results with related literature and presented the main of our finding.

It has been shown through statistics that even if most of organizations are aware of the strengths and benefits of software reuse, they don't consider reuse as part of the company's culture, and most of them didn't implement efficient software reuse programs and not apply the most effective reuse strategies, methods and practices.
\end{abstract}

Key words: Moroccan software industry, organization, survey, software reuse.

\section{Introduction}

Software reuse is a process aiming to define a set of systematic operating procedures to specify, produce, classify, retrieve, and adapt software artifacts for creating software systems from them [1]. The reuse community [2]-[34], [35], [23] agrees that a systematic reuse offers a great potential of significant gains for organizations by reducing costs and efforts, and accelerating time to market of software products [2].

Organizations try to adopt software reuse because of its potential effect on competitiveness, but they need answers to practical questions. However, there is a high up-front risk of reuse adoption, because a significant initial organization investment is required, while the ROI is not guaranteed [16]. Several Software Reuse Models have been suggested through literature to face the reuse adoption problem [16], [17] 
[2], [34], [35], [36]. Furthermore, it is difficult to determine exactly what makes a reuse program successful: it could be the organization structure and size, the organization's experience in the field, technical issues, financial issues...

To find these answers and address the way of reuse adoption in different organizations and their various aspects in the Moroccan software industry context, we conducted a survey of 84 technical software participants with different backgrounds: software managers, analysts, engineers and software developers, in different types of organizations: International IT service provider, public institutions, semi-public institution (All respondents work in Morocco).

The aim of the survey is to evaluate in practice the adoption of techniques, methods and practices proposed through literature, and highlight the motivations as well as difficulties to implement a successful reuse program by different types of organizations in Morocco.

The paper presents a complete survey method, including the process, data collection, and analysis phases, as well as a discussion on the study's validity...

The survey consisted of 28 questions, grouped into 4 sections and backed up our findings with statistics. A discussion was also given based on the comparison of the survey results with related literature and presented the main of our finding.

The remainder of this paper is organized as follows: Section 2 presents the related works, whereas section 3 describes our survey and the method used, results are presented in section 4 . Section 5 presents a discussion of our findings and overviews a statistical analysis, and section 6 contains the conclusion of our analysis.

\section{Related Works}

Few studies were interested in the industrial application of reuse. An analysis of 25 software projects from a NASA software development environment that actively reuses software was done by [3]. This analysis highlights that software reuse rate reaches $32 \%$ per project. The aforementioned level of reuse was achieved by applying many strategies like product lines, design patterns and architecture-based code generation. The analysis also concludes that significant economic benefits can be realized through the systematic application of reuse, because of the little development efforts expending. All in all, the analysis in itself was interesting. However, it focused solely on the reusing of source code.

Frakes and Fox [24] conducted an empirical study in 28 software organizations, presenting 16 questions about software reuse. The aim of their study was to find some practical answers for software organizations that are implementing software reuse programs. Some factors influencing software reuse are presented through this study.

Another study was performed by [4] using qualitative and quantitative data from six open source software projects, to explore practices of code reuse in open source software development and discuss the different aspects of software reusing. The analysis distinguishes two types of code reuse: architectural reuse and functional reuse and was essentially about the importance of reusable software components.

A survey of 12 industrial developers working at different organizations ( 6 companies) was conducted by [5], to evaluate questions about pragmatic reuse. The survey investigated how developers plan and perform software reuse tasks, but without providing case studies investigating the effectiveness of the presented approaches compared to the current developer practices.

Another survey of 59 industrial software developers was conducted by [6] to address the frequency, motivation difficulties and execution of pragmatic reuse practices in the industrial development experience. That survey consisted of 10 questions only, but by using more questions the results would be better and more significant. Also, this study was interested in pragmatic reuse i.e. reuse of source code and not about 
the whole process of software reuse.

After reviewing the literature, it's far applicable to say that none of these studies satisfies our desire to identify the current state of software reuse and the way it's adopted in different organizations inside the Moroccan software industry context. These studies are interesting but did not present large results and did not target a large specter of profiles (developers, managers, technical expert, software architecture...). Most of these observational studies, are limited at the reuse of source code and did not take interest in the other activities of software development life cycle. At the same time, no study focused on software reusing in Moroccan organizations.

\section{Method}

A survey is a research method and strategy aiming to collect and summarize information from a large population [7]. This information is used in order to describe, explain and measure behavior or attitude [8].

There are three types of surveys [9]: Descriptive, explanatory, and exploratory surveys.

- Descriptive surveys allow assertions about some population with the focus on what occurred rather than why [8].

- Explanatory surveys are used in order to make explanatory affirmations about the population.

- Exploratory surveys are considered as a pre-study to find out new opportunities and risks for a more thorough investigation in the future.

In our case we conducted a descriptive survey [10] to study how Software reuse is addressed with practical experience in the software industry.

\subsection{The Survey Process}

To perform this survey, and ensure the accuracy of our study, we applied the process proposed by [11] based on the following 6 steps (see Figure 1) and using activities of a survey process as described by [10]:

- Survey definition: in this first step in the survey we defined the goal of the study [12], and clearly depicts the topic, context, and scope of survey.

- Survey design: we presented the survey plan by defining a several activities and decisions. We exposed the survey goal into research questions. From these questions, we designed an online questionnaire as data collection instrument. Furthermore, we validated the data collection instrument.

- Survey implementation: based on the study design, we make the survey executable with scheduling the survey and setting up an online infrastructure to collect the data.

- Survey execution: we run the survey according to the survey plan and collect the required data.

- Survey analysis: we analyzed and interpreted the collected data and check data validity based on information from design and execution.

- Survey packaging: report the survey and its result to disseminate and share results with external parties.

Details of these steps are discussed in the following sub-sections.

These steps are performed in an iterative way. In the Fig. 1 we illustrate the survey process: boxes present the process steps; arrows around the boxes show typical iterations in the process.

\subsection{Survey Definition}

The survey goal was to capture how organizations practice software reuse, their motivations, the difficulties they face and the frequency of this reuse in a practical context.

We based our survey on the conceptual model, on existing established and documented surveys [7, 11, 15]. 


\subsection{Survey Design}

Definition of the target population and the survey sample. We have conducted a systematic survey for a wide range of information system domains including software engineering, support, infrastructure, telecommunication, etc. The results came from various companies and kind of organizations in Morocco (International and Moroccan IT service provider, public institutions, semi-public institutions...). This survey opted to include various technical skills and exclude practitioner opinion, when this practitioner did not have a technical profile (Project Manager, Technical expert, Developer... are considered as technical profile).

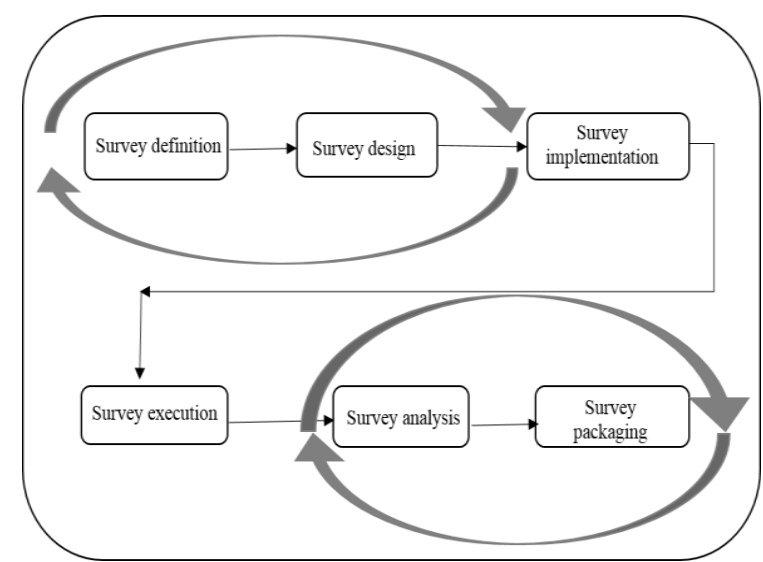

Fig. 1. Survey process overview according to [11].

We identified an initial group of practitioners using mailing lists related to their respective companies. Additional groups were enabled based on initial practitioners. The survey was anonymous. A various companies and organizations were considered, and we received 84 responses.

The survey was first opened on August 2017 and closed on November 2017.

Approach for data collection. Multiple methods of data collection are used to gather information from the target population [11].

We have conducted this empirical study by using on-line questionnaires through Google forms [14]. The advantage of this tool is that it is easy for use, popular and suitable for surveys. In addition, there is no limitation on the number of questions.

Questionnaire design: questions. We relied on our previous works [2] and our experience in an international IT service provider organization to identify questions for this survey. The survey consisted of 28 questions, grouped into 4 sections:

- About your organization: in this section we have 2 questions about the respondent's organization.

- Your personal information: in this section

- 2 questions are designed to address the personal information of respondent.

- Reuse in your organization: this section presents 17 questions concerning how organizations encourage software reuse.

- Your experience with reuse: this section exposes 7 questions to help respondent sharing his experience on reuse if existed.

Validity issues. The most important purpose of validity issues is to reduce the likelihood of bias. According to [7] validity issues have to be defined through internal and external validity. Internal validity dealt with theoretic issues and external validity focus on issues revealed during survey implementation and execution.

In our context, all survey design attributes are reviewed and evaluated by experts in order to define and resolve all possible internal issues. External validity should be based on findings. We have therefore 
established that the practitioners must have a technical diploma and operate in one of the information system fields despite their activity field. This study did not take into consideration respondents without an employment status. For duplicate responses, we retain the most complete one.

\subsection{Survey Implementation}

As mentioned in [11], the objective of the survey implementation step is to prepare and produce all the material needed in order to effectively meet the survey objectives. We have indeed performed a pilot survey to evaluate the questionnaire before using it on the large scale.

\subsection{Survey Execution}

The objective of the execution step is to run the survey and collect the required data. According to [13], we illustrate in table 1 several survey execution elements adapting to our context

Table 1. The Survey Execution Elements

\begin{tabular}{ll}
\hline Element & Description \\
\hline Media & $\begin{array}{l}\text { The survey was mediated } \\
\text { through mail }\end{array}$ \\
Execution time & Three weeks \\
Response burden & Seven months \\
Follow-up & $\begin{array}{l}\text { To encourage response and } \\
\text { prevent non-response, we } \\
\text { procedures }\end{array}$ \\
& $\begin{array}{l}\text { based mainly on reactivate } \\
\text { responders through mail }\end{array}$ \\
& 84 responses received \\
\hline \hline
\end{tabular}

\subsection{Survey Packaging}

The objective of the packaging step is to report the survey and its result. In our context, the key target groups are: Management, researchers in software reuse and researchers who want to conduct a survey. We reported specific and targeted report results (section 5) for each of these target groups according to guidelines and best practices described in [11].

\section{Results}

A total of 87 respondents participated in the survey. We discarded responses from three respondents. First, we removed a duplicated response informed by the same respondent. Then 2 responses coming from respondents that do not possess a technical profile were also discarded, because we were only interested by technical profiles in this survey.

Finally, only 84 responses are retained and are considered in our statistical analysis. ([37] can be checked for more details)

Q1: What is the type of your organization?

International and Moroccan private institution: 22\%. Moroccan Public institution: 20\%. Moroccan Semi-public institution: 8\%. International and Moroccan IT service provider: $49 \%$.

Q2: What is the size of your organization?

Less than 10 employees: 4\%. From 10 to 250 employees: 39\%. From 251 to 5000 employees: 44\%. More than 5000 employees: $14 \%$.

Q3: What is your activity field?

Respondents are from different activity fields as, computer development, finance, telecom... but most of 
them are from IT organizations.

Q4: What is your current employment status? What is your degree and How many years of experience have you accumulated in the computer field?

Different answers are given for this question, but what we can noted is that respondents have different status: managers, analysts, engineers and developers, and have between 1 and 23 years of experience

Q5. Does your organization encourage software reuse?

Yes: $39 \%$. No: $18 \%$. Partially: $43 \%$.

Q6. Does your organization make you aware of the benefits of software reuse?

No: $55 \%$. Yes: $45 \%$.

Q7. Is there a responsible for reuse in the organization?

No: $62 \%$. Yes: $35 \%$. I don't know: $2 \%$.

Q8. If yes, what is his position within the organization?

Project Manager: 42\%. Technical expert: 39\%. Engineer (developer): 14\%. No one: 3\%. I don't know: 3\%.

Q9. Is training scheduled for employees to encourage re-use?

$80 \%$ of respondents have said that there is no reuse training or programme in their organization.

Q10. How are employees who practice reuse recognized in your organization?

I don't know: 1\%. Better evolution: $1 \%$.

No recognition: 64\%. Financial Recognition: 4\%. Moral Recognition: 30\%.

Q11. Does the organization have a common repository to store reusable assets or components?

No: $44 \%$. Yes: $54 \%$. I don't know: $2 \%$.

Q12. If so, are the various employees sufficiently informed of its existence?

All: $20 \%$. The majority: $27 \%$. A minority: $53 \%$.

Q13. Does the organization have a guide for setting up reuse?

No: $80 \%$. Yes: $14 \%$. I don't know: $6 \%$.

Q14. At what stage of the project, is reuse practiced?

Different stages are given by respondents: it varies from the design phase to the recipe phase.

Q15. What are the types of reusable components in your organization?

Different respondents are given: design, code, documents, framework...

Q16. Does the organization have a model project and/or project type collection reused at the input of a new project?

No: $58 \%$. Yes: $40 \%$. In the process of setting up:1\%. I don't know: $1 \%$.

Q17. What is the frequency of reuse in projects?

Never: 7\%. Rare: 30\%. Average: 50\%. Systematic: 13\%.

Q18. If there is a team dedicated to re-use, how many employees are there?

$45 \%$ of respondents confirmed that they didn't have a reuse dedicated team in their organization.

Q19. How much reuse is possible in your projects?

$70 \%$ of respondents confirmed that the percentage of possible reuse in their projects is less than $50 \%$.

Q20. What is the re-used part of your projects?

Null: 15\%. Less than 50\%: 77\%. More than 50\%: 9\%.

Q21: Is there a distinction between a team or collaborators producing reusable components and entity / collaborators consuming reusable components?

No: $74 \%$. Yes: $25 \%$. I don't know: $1 \%$.

Q22: Have you ever produced a reusable component?

No: $27 \%$. Yes: $73 \%$.

Q23. Is your practice of software reuse a personal initiative? 
No: $40 \%$. Yes: $60 \%$.

Q24. If so, what is your motivation?

Part of your organization's best practices: 29\%. Personal initiative: 55\%. Goal set with your organization: $16 \%$.

Q25. If your profile is project manager, have you encouraged the production of a reusable component on one of your projects?

$80 \%$ of project managers participating in the survey encourage the production of a reusable component on one of your projects.

Q26. If yes, is the charge for producing a reusable component deducted from the project budget or from your organization?

Most of answers indicate that the production of reusable components is deducted from the project budget.

Q27. In your opinion, what are the barriers for reuse?

A lot of answers are given for this question, but they are mainly related to three aspects: Organizational issues, Technological issues and Cultural issues.

Q28. What will encourage you to consume reusable components?

A lot of answers are given for this question, but they are mainly about reducing efforts, time and costs.

\section{Discussion}

In this section, we discuss and interpret the results of our survey, with some explanations provided by industry professionals. Then, we examine whether our findings are confirmed by the literature.

Results issues are analyzed using the quantitative analysis which is an inductive research approach, i.e., from specific observations it is possible to extract larger generalizations and theories [13].

We will examine some reuse characteristics or factors that influence the success of software reuse in the industrial context. Characteristics are organized in three perspectives: Organizational, technological, and cultural (provided by the survey responses). This division is useful for organizations that need to focus on one perspective at a time when adopting reuse.

We structured this paragraph as following: The finding interpretation (i.e. allows to present the survey finding and our interpretation), related literature (i.e. presents the position found in literature) and results (provides a result of the comparison of our finding with related literature).

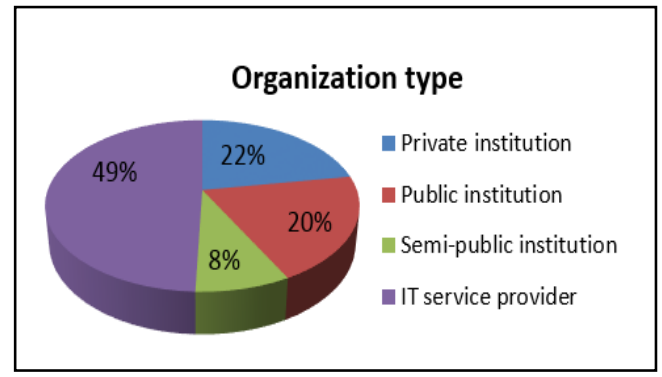

Fig. 2. Organization type.

\subsection{Organizational Perspectives}

Organizational perspectives refer to reuse characteristics or factors relate to organizational issues as structure, size type, reuse activities...

Finding Interpretation: It turned out that the respondents came from quite mixed environments, but about $49 \%$ of respondents were from "IT service provider" international and Moroccan. (Fig. 2). Also, 
organizations of all sizes participated, from very small (1 - 10 employees) to very large (more than 5000 employees), but most respondents were from small and medium organizations (Fig. 3). Organizations as IT service provider or private can be international or Moroccan, but they are all located in Morocco.

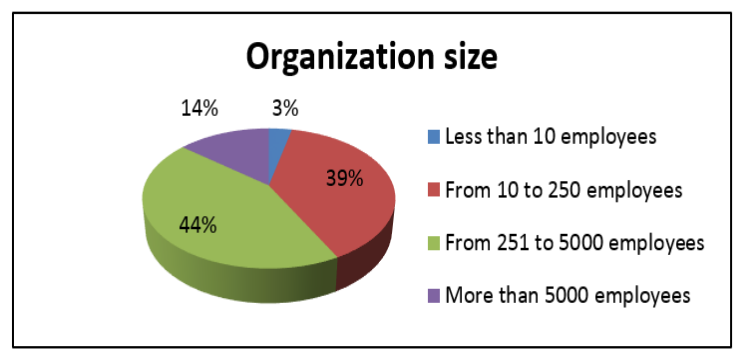

Fig. 3. Organization size.

We notice that that $39 \%$ of organizations totally encourage the software reuse and $43 \%$ partially does so (Fig. 4). That's means that many organizations are aware of reuse benefits, specially IT service provider organizations and private institutions. This behavior is illustrated in the Fig. 5 which represents frequency of reuse by Organization Type, which is about $50 \%$.

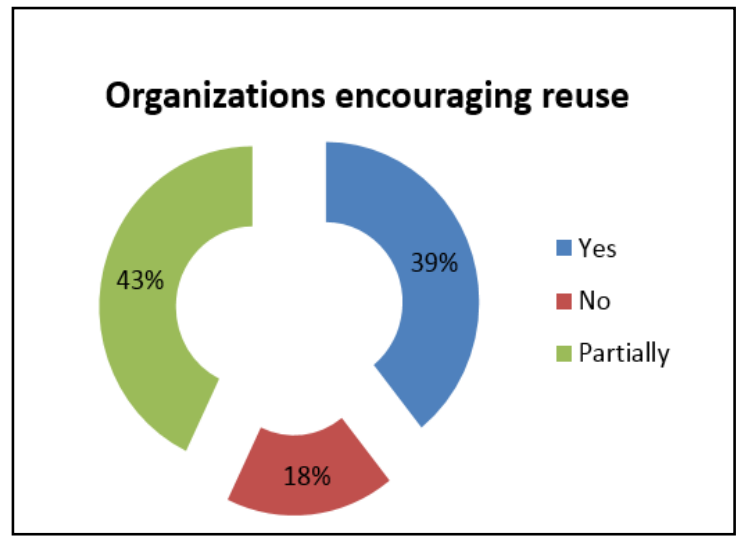

Fig. 4. Organizations encouraging reuse.

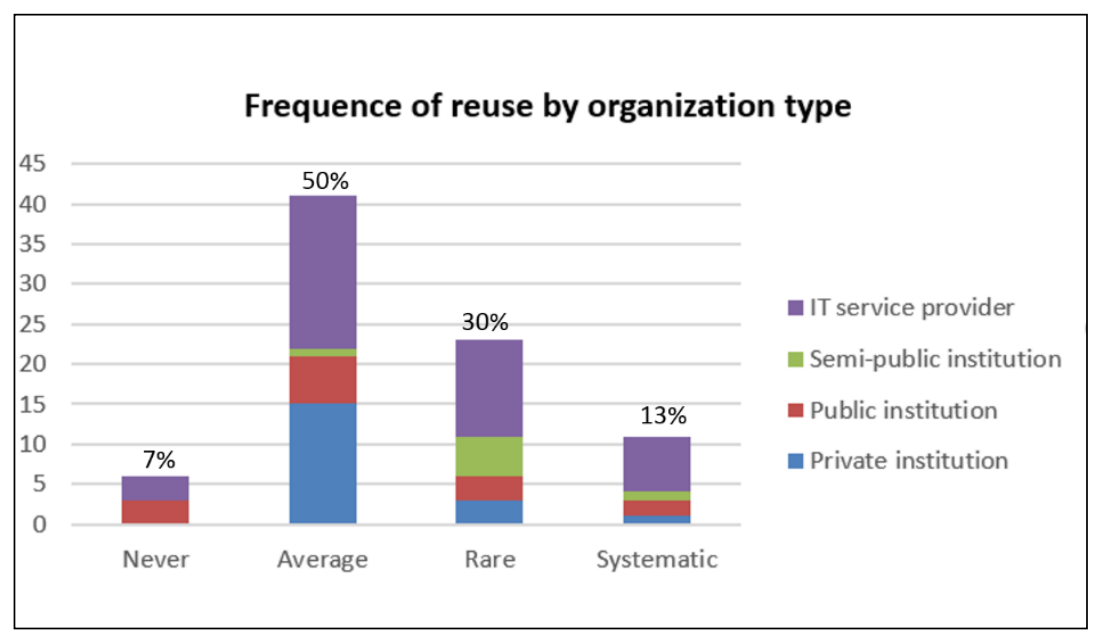

Fig. 5. Frequency of reuse by organization type.

Even if many organizations encourage totally or partially the software reuse, most of them don't have any 
guide for setting up the reuse process the correct way. Fig. 6 presents the availability of a Reuse Implementation Guide in organizations. The chart shows that there are only $14 \%$ of organizations having a guide to implement software reuse (even if $82 \%$ of them encourage software reusing).

So according to our results we can state that the Reuse process remains implicit in these organizations.

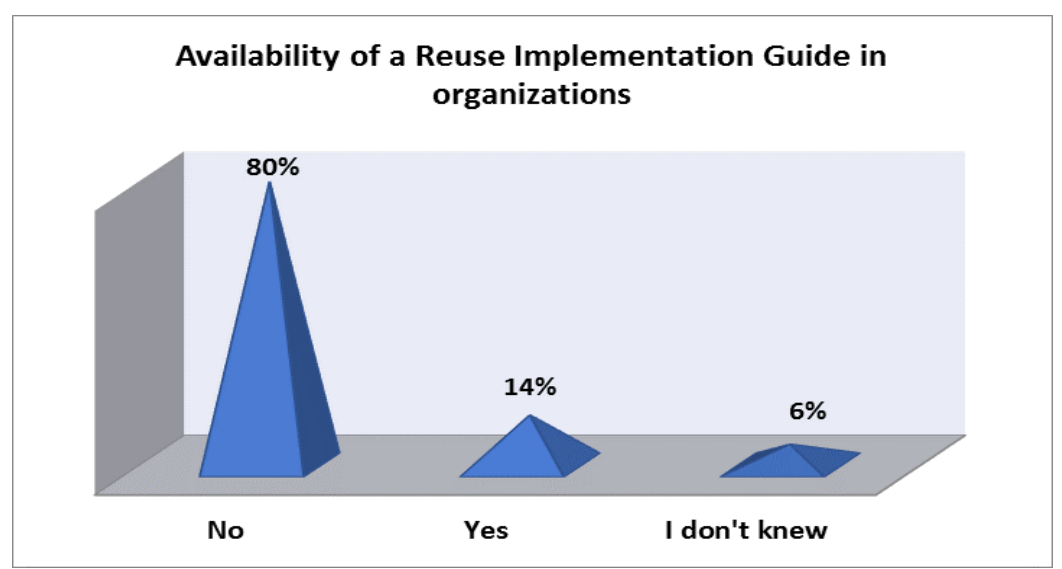

Fig. 6. Availability of reuse implementation guide in organizations.

An explanation of these behaviors would be that reuse is well perceived by It Provider organizations and private organizations than other organizations types, because they are more aware about benefits of reuse in improving time-to-market and its potential effect on competitiveness, while semi-public and public institutions are not taking seriously the initiative of promoting reuse. However even if organizations are aware of benefits of reuse, most of them have no strategy or guide for implementing a systematic reuse in their development process.

Related literature: The literature considered reuse only in software development organizations and address the importance of investing in software reuse to improve productivity and quality [22], with planning or developing a reuse program. Unfortunately, there is still not enough data available in industrial experience about software reuse models to be adopted by organizations, the existing reuse models are only from literature [24], [2], [20].

According to [23], the size of organization is not a factor that influences directly software reuse, but a guide for promoting reuse is very needed for each type and size of organization. The necessity of using a guide for promoting software reuse in organization is also reported by [20], [27], [28].

\section{Results:}

- Reuse success is possible in any type or size of organizations; this finding is also confirmed by literature.

- Previous works highlighted the necessity of defining a specific reuse guide or reuse model to aid an organization to implement a success reuse. However, this is not the case for most of organizations in this survey.

- Most of organizations that participated in the survey, declare that reuse is not easy to be adopted, because it introduces changes in the organization's culture and process. Same is also supported by literature.

\subsection{Technological Perspectives}

Technological perspectives refer to reuse characteristics or factors that describe how technology can support a reuse program.

Finding Interpretation. The achievement of software reuse is related to the employment of reusable 
assets or components [20]. Assets can be a source code, documentation, requirement, architecture, application design, test...Through this survey we would like to know how reuse is adopted in organizations and if they produce enough reusable assets or not. For this we have stood out the percentage of production of reusable components or assets in each organization using the chart in Fig. 7. The chart shows that $73 \%$ of asked organizations are producing the reusable assets and only $27 \%$ doses not.

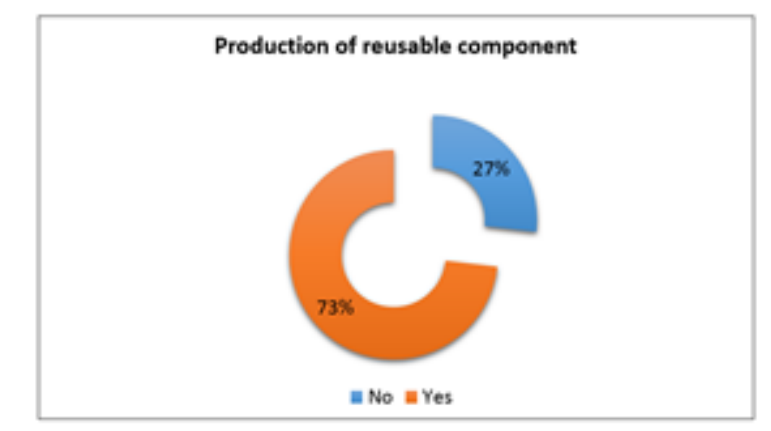

Fig. 7. Production of reusable component.

It seems that the percentage of producing reusable components is important, thereby we need to know: if there is any repository for storing these assets and components.

Reusable assets and components needs to be collected and organized into a central repository for a large use by different developers in the organization. According to the Fig. 8, 54\% of organizations have a central repository, however no details about retrieving process or using these assets are available.

Through this survey we need to know if organizations believe that source code is the only asset that can be reused, or if there are other kind of assets. Said assets were categorized into four reusable components type: code (for source code), conception (for architecture, application design...), framework (for tools and platforms), and documentation (for requirements specification, test documentation, project plans, technical documentation...). Fig. 9 presents the reusable component in different type of organization according to their type. We can see from this graph that the most used type of assets in different type of organizations is the source code, then documentation, then Framework and conception.

We can also say that service provider and private organizations are more interesting on the reusable assets than other organization type.

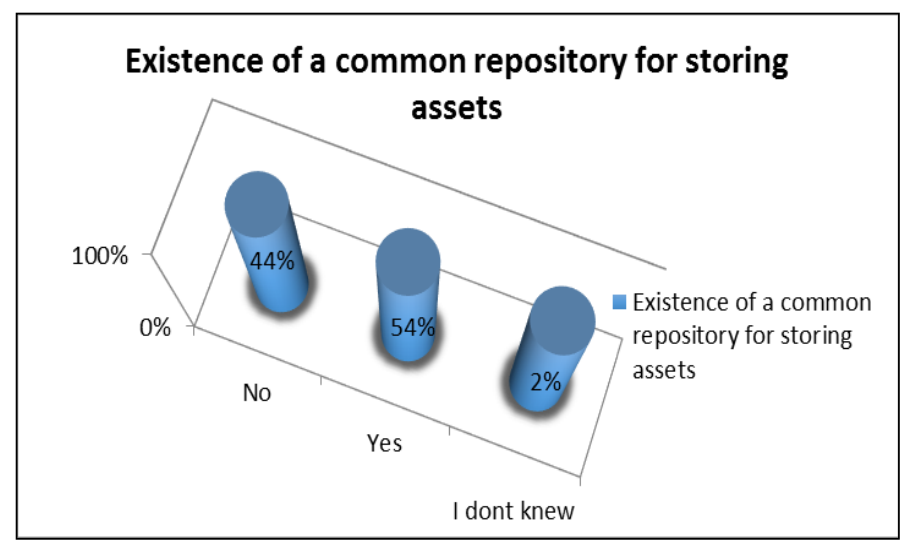

Fig. 8. Existence of a common repository for storing assets.

This can be explained, by the organization culture which believes that reuse approach is related to reuse of source code, that's why this kind of asset is the most used. Documentation is also much used because its 
can be simply produced by adapting existing documents with a small effort, and it's the case also for frameworks, especially for organizations working on a specific domain, so they can adapt easily assets for other similar projects.

Reusable assets can also be built from scratch with a maximum of abstraction, so they can be used in different projects. However, this process is expensive, and most industrial organizations neither have the budget or the time for this kind of investment.

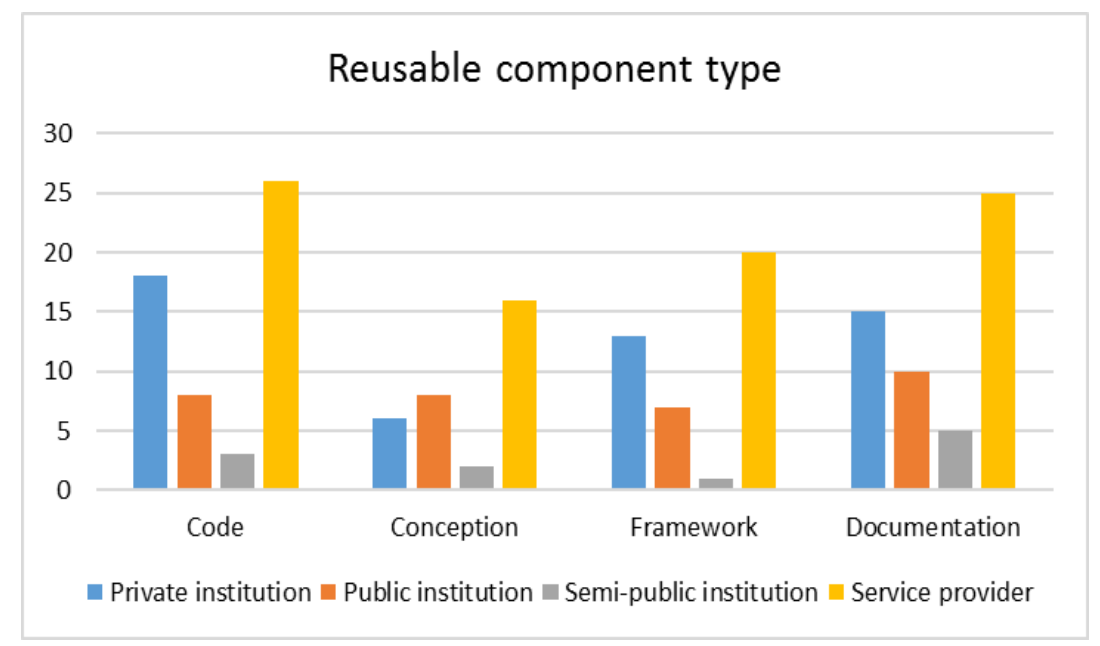

Fig. 9. Reusable component type.

Related literature: Literature highlighted a clear predominance of reuse of source code in organizations in detriment of reuse of other reusable assets [27], [30]. The construction and integration of reusable software components contribute to achieve a reuse success [27], [2], [31]. However, there is an additional cost when developing software with reusable components, even if this cost is negligible when reusing these components on other projects [20]. The lack of reusing components and producing reusable assets is due to the conflict with team priorities that should respect project time and budget [32]. For maintaining the catalog of reusable components, a repository with retrieval mechanisms is necessary for assisting developers on selecting the appropriate software components [20], [24], [27].

\section{Results:}

- Literature indicates that software reuse should be considered during all the development life-cycle and not only in the implementation step. But, according to our finding, reuse is most considered in development step only.

- Previous works insist on promoting reuse of all types of assets, and not only source code, while our finding raises that it is the most used type in the participating organizations.

- A repository with a well-defined retrieval mechanism is needed for achieving a success reuse as mentioned in literature. However, only a minority of organizations stated that they have a repository for storing reusable assets, but without any retrieval mechanism.

\subsection{Cultural Perspectives}

Cultural perspectives refer to reuse characteristics or factors related to individuals involved in software reuse.

Finding Interpretation. It appeared in this survey that we have a large activity field and not only a pure IT field (like Banking and Insurance, Finance, Telecom...). Respondents are from different field, but all of them are industrial software profiles with various status (Project Manager, Technical expert, Developer...) and had various years of experience (from 1 year to 22 years). 
According to the Bar Chart in the Fig. 10; 55\% of respondents produce reusable components by their own initiative, and only $29 \%$ of them because it's a part of organization best practices. We observed also in our survey that most of organizations don't plan any training programs for employees to promote reuse.

First of all, questioned organizations in this survey are not challenging employees to practice systematic reuse, and secondly, they didn't integrated reuse as a part of best practices in their production process. However, if organizations wish to progress, they should not be limited to the personal initiative of their employees. They ought to implement efficient software reuse programs. This fact is confirmed by the chart in the Fig. 11, which shows that there is about $64 \%$ of respondents ensures that no recognition due to the practice of reuse is made, and about $30 \%$ are morally encouraged.

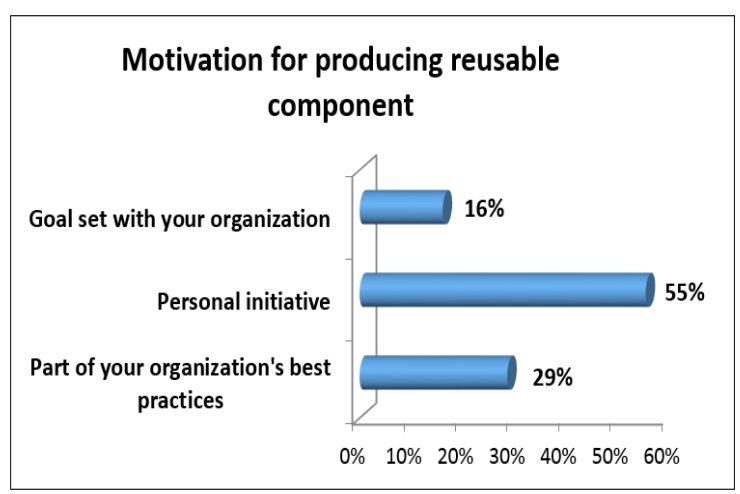

Fig. 10. Motivation for producing reusable component.

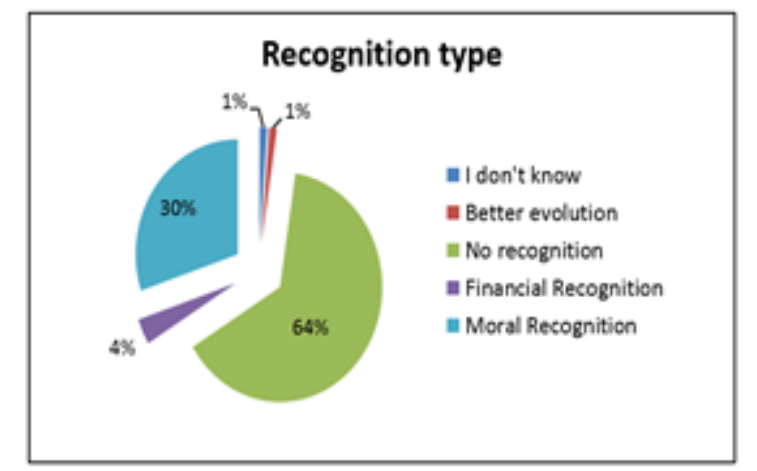

Fig. 11. Organization recognition type.

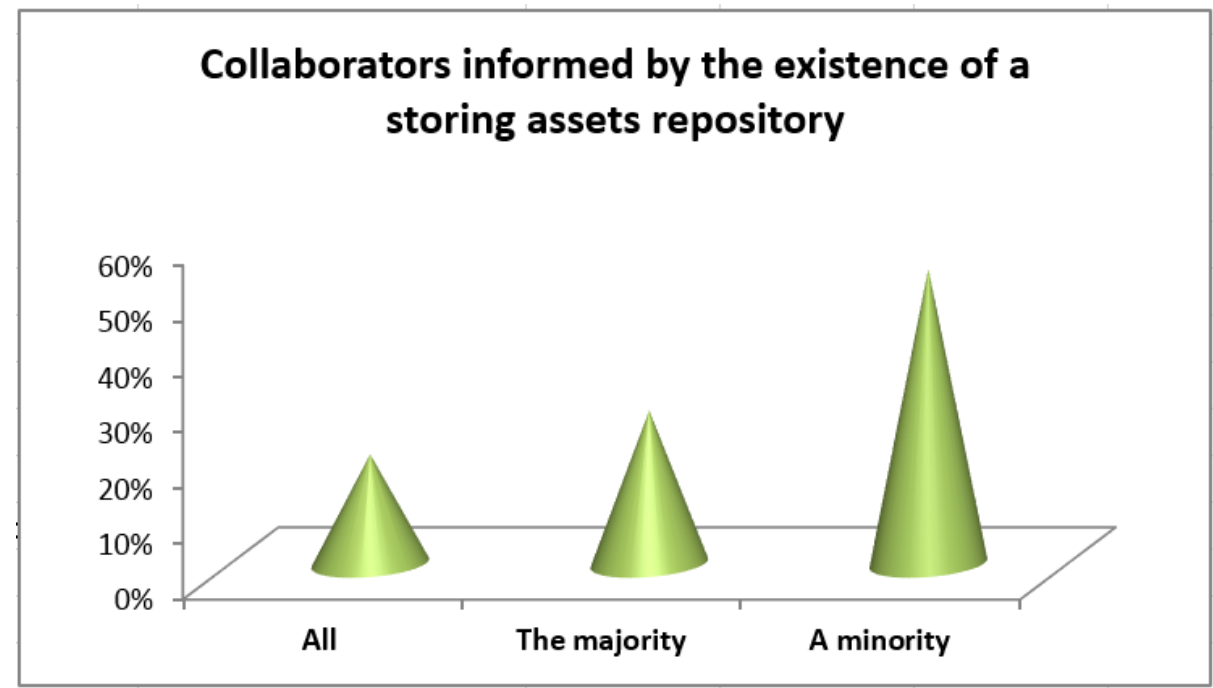

Fig. 12. Collaborators informed by the existence of a storing assets repository. 
Through our survey we can notice that employees are not involved in software reuse practices, and there are no assigned teams for software reuse to build reusable assets and communicate them to the concerned parties. The existence of an independent development team dedicated for reuse activities have some influence on reuse success.

Also, employees are not informed by the existence of repository for storing assets, if exist (about 60\%).

Related literature: Some researches, such as [23], [24], stated that few organizations are adopting a reuse education program, even if reuse education have an influence on the reuse success. The author in [23], highlighted on his survey that organizations didn't adopt any training programs related to reuse.

The implementation of reuse education programs (reuse culture) in organizations is also reported by [24], [25], [26], [27] as an influencing factor for achieving reuse.

For [27] in smaller team, reuse is carried out by an individual effort by asking colleagues form source code. In medium teams the individuals have more freedom to take the initiative of promoting reuse. Hence personal communication is crucial in organization with the goal of clarification and sharing [32].

To obtain good results with reuse, [23] stated that existence of an independent development team dedicated for reuse activities have some influence on reuse success.

Anshul [33] pointed out that organizations don't reuse because of the factors relating to corporate culture. Author in his work highlighted the necessity of defining new roles, and responsibilities for better implementation of software reuse in the organization.

\section{Results:}

- Organizations didn't have a separate team dedicated for promoting software reuse: this observation is not aligned with literature recommendation for obtaining reuse benefits.

- According to our survey's results, the reuse success in organizations is due to individual efforts and not to organizational process. Literature contrasts our finding, and consider that systematic reuse process is a strong factor influencing reuse in organizations.

- There is no training plan for employees to promote reuse in most organizations: our finding agrees with literature which states that training is not one of the most required activities for a successful reuse.

- In most organizations, reuse is not part of the company's culture, which makes the adoption of reuse very complex. Literature highlighted that reuse's success is tightly coupled with the organization's culture.

\section{Conclusions}

Recently there is a major need for organizations of any kind (It service provider, private or public institution...) to reinforce reuse practices and processes, starting from a "functional reuse" and going towards a "technical reuse".

We conducted this survey to capture how organizations among the Moroccan software industry context practice software reuse, their motivations, the difficulties they encounter and the frequency of this reuse in practice.

We received responses from 84 various technical profiles from a wide range of organization sizes and types implanted in Morocco. A qualitative analysis of respondents was performed to analyze and discuss our results.

We have shown through statistics that even if most of the organizations are aware of the strengths and benefits of software reuse, they don't consider reuse as part of the company's culture, and they don't encourage reuse practices enough through teaching their individuals how to practice software reusing. Furthermore, we have pointed out that most of the organizations haven't implemented efficient software reuse programs and they didn't apply the most effective reuse strategies, methods and practices. Without a 
holistic approach towards software reuse, they are not able to identify potential improvement areas for achieving even greater benefits.

Through this survey we can conclude that the reuse adoption concepts need to be improved and streamlined inside the Moroccan software industry context. However, establishing any kind of systematic reuse poses significant challenges and requires a structured decisional support. Further work is needed to support organizations on identifying and installing the required best practices and approaches for achieving reuse in their specific context.

\section{References}

[1] Buglione. L, Lami, V. W. G., Von, W. C. G, Mc, C. F., \& Rossa, H. J. C. (2013). Leveraging reuse-related maturity issues for achieving higher maturity and capability levels. Safe and Secure Software Reuse.

[2] Bauer, V. et al. (2016). Comparing reuse practices in two large software-producing companies. Journal of Systems and Software, 117.

[3] Selby. R. W. (2005). Enabling reuse-based software development of large-scale systems. IEEE Trans. Softw. Eng., 31(6), 495-510.

[4] Haefliger, S., Von, K. G., \& Spaeth, S, (2008). Code reuse in open source software. Manage. Sci., 54(1), 180-193.

[5] Holmes, R., \& Walker. R. J. (2007). Supporting the investigation and planning of pragmatic reuse tasks. Proceedings of the IEEE Int. Conf. Softw. Eng.

[6] Walker, R., \& Cottrell, R. (2016). Pragmatic software reuse: A view from the trenches. Science.

[7] Molléri, J. S., Petersen, K., \& Mendes, E. (2016). Survey guidelines in software engineering: An Annotated review. Proceedings of the 10th ACM/IEEE International Symposium on Empirical Software Engineering and Measurement.

[8] Gray, D. E. (2009). Doing Research in the Real World. London: Sage.

[9] Wohlin, C., Hoest, M., \& Henningsson, K. (2003). Empricial research methods in software engineering. Empirical Methods and Studies in Software Engineering (pp. 7-23).

[10] Kitchenham, B. A., Pfleeger, S. L., Pickard, L. M., Jones, P. W., Hoaglin, D. C., Emam, K. E., \& Rosenberg, J. (2002). Preliminary Guidelines for Empirical Research in Software Engineering. IEEE Transactions on Software Engineering.

[11] Ciolkowski, M., Laitenberger, O., Vegas, S., \& Biffl, S. (2003). Practical experiences in the design and conduct of surveys in empirical software engineering.

[12] Freimut, B., Punter, T., Biffl, S., \& Ciolkowski, M. (2002). State-of-the-art in empirical studies.

[13] Linaker, J., Sulaman, S. M., Höst, M., \& De Mello, R. M. (2015). Guidelines for conducting surveys in software engineering v. 1.1.

[14] Forms.

https://docs.google.com/forms/d/e/1FAIpQLSdeOJoqKQ2AJAE6NX-kZRkBl4kvXSLL-By3uad_zvSoNhN $\mathrm{keQ} /$ viewform

[15] Ameller, D., Galster, M., Avgeriou, P., \& Franch, X. (2016). A new reuse capability and maturity model: An overview. Proceedings of the 2018 International Conference on Software Engineering and Information Management.

[16] Younoussi, S., \& Roudies, O. (2018). Towards a new reuse capability and maturity model. Proceedings of the 11th Iadis International Conference Information Systems.

[17] Srivastava, D. K., \& Nair, P. (2017). An extensive conception of reusability in software component engineering. Proceedings of International Conference on Communication and Networks (pp. 449-457). Springer, Singapore. 
[18] Younoussi, S., \& Roudies, O. (2016, May). Capability and maturity model for Reuse: A comparative study. Proceedings of the 2016 2nd International Conference on In Cloud Computing Technologies and Applications (CloudTech).

[19] Garcia, V., Lucrédio, D., \& Alvaro, A. (2007). Towards a maturity model for a reuse incremental adoption. Proceedings of Simpósio Brasileiro de Componentes, Arquitetura e Reutilização de Software (SBCARS).

[20] LucrÉdio, D., Dos, S. B., Kellyton, A. (2008). Software reuse: The Brazilian industry scenario. Journal of Systems and Software, 81(6), 996-1013.

[21] Frakes, W. B., \& Fox, C. J. (1995). Sixteen questions about software reuse. Communications of the ACM.

[22] Morisio, M., Ezran, M., \& Tully, C. (2002). Success and failure factors in software reuse. IEEE Transactions on Software Engineering.

[23] Sherif, K., \& Vinze, A. (2003). Barriers to adoption of software reuse: A qualitative study. Information \& Management, 41(2), 159-175.

[24] Bombonatti, D., \& Goulão, M., Synergies and tradeoffs in software reuse - A systematic mapping study. Software: Practice and Experience.

[25] Rothenberger, M. A. (2003). Project-level reuse factors: Drivers for variation within software development environments. Decision Sciences, 34(1), 83-106.

[26] Rine, D. C., \& Nada, N. (2000). Three empirical studies of a software reuse reference model. Software: Practice and Experience.

[27] Konda, B. M., \& Mandava, K. K. (2010). A systematic mapping study on software reuse. master's thesis, Blekinge Institute of Technology, School of Computing, Sweden.

[28] Joos, R. (1994). Software reuse at motorola. Software, 11(5), 42-47.

[29] Younoussi, S., \& Roudies, O. (2015). All about software reusability: A systematic literature review. Journal of Theoretical and Applied Information Technology.

[30] Anshul, K., \& Sumesh, S. (2017). A comparison based framework for reuse adoption in an organization. Indian Journal of Computer Science and Engineering .

[31] Davis, M. (1992). Stars reuse maturity model: Guidelines for reuse strategy formulation. Proceedings of the Fifth Annual Workshop on Institutionalizing Software Reuse.

[32] Davis, T. (1993). The reuse capability model: A basis for improving an organization's reuse capability. Proceedings of the 2nd International Workshop on Software Reuse. Lucca, Italy.

[33] Spoelstra, W. (2010). Reusing software assets in agile development organizations-a management tool. Retrieved from: web site http://essay.utwente.nl/59917/1/MA_thesis_W_Spoelstra.pdf accessed on $30 / 03 / 2015$

[34] Spreadsheets.

Retrieved

from: https://docs.google.com/spreadsheets/d/16Y1zHRylqL6uG5E48ecQ2SftBujtf20vLSVi6Uc490g/edit\#g id $=1348654971$

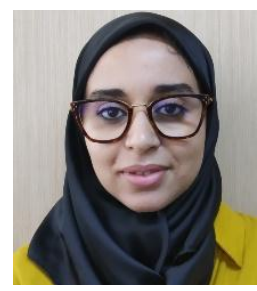

Siham Younoussi was born in Rabat, Morocco. She is computer science engineer from "Institut National de Statistique et d'Economie Appliqué (INSEA) in 2008, and a member of the SIWEB Research Team since 2013. Currently, she is an IT project manager. She is currently working towards the Ph.D. degree in Ecole Mohammadia d'Ingénieurs (EMI), Mohammed V University in Rabat-Morocco. She is interested in software reuse and Software product lines. 